\title{
Association of abnormal hind-limb postures and back arch with gait abnormality in dairy cattle
}

\author{
A. C. Hoffman, ${ }^{\star}$ D. A. Moore, ${ }^{\star 1}$ J. Vanegas,, and J. R. Wenz ${ }^{\star}$ \\ *Department of Veterinary Clinical Sciences, College of Veterinary Medicine, Washington State University, Pullman 99164 \\ †Department of Clinical Sciences, College of Veterinary Medicine, Oregon State University, Corvallis 97331
}

\begin{abstract}
Detection of lameness in individual cows is important for the prompt treatment of this painful and production-limiting disease. Current methods for lameness detection involve watching cows walk for several strides. If clinical signs predictive of lameness could be observed more conveniently, as cows are undergoing regularly scheduled examinations while standing, detection levels could increase. The objective of this study was to assess the association between postures observed while cows are standing in stanchions and clinical lameness evaluated by locomotion scoring, and to evaluate the observation of these postures as a test for lameness. The study included 1,243 cows from 4 farms. Cows were observed while standing in stanchions for regularly scheduled management procedures and the presence of arched back and cow-hocked, widestance, and favored-limb postures were recorded. The same cows were locomotion-scored as they exited the milking parlor. The proportion of cows observed with arched back and cow-hocked and favored-limb postures increased with increasing severity of lameness (higher locomotion score) but did not increase for the widestance posture. For the presence of these postures as a test for lameness (locomotion score $\geq 3$ ), sensitivity and specificity were 0.63 and 0.64 for back arch, 0.54 and 0.57 for cow hocks, and 0.05 and 0.98 for favored limb. Back-arched, cow-hocked, and favored limb postures were associated with lameness but were not highly sensitive or specific as diagnostic tests. However, observation of back arch may be useful to identify cows needing further examination.
\end{abstract}

Key words: back arch, lameness, cow hocked

\section{INTRODUCTION}

Lameness is an important problem in US dairy herds for both animal welfare (O'Callaghan et al., 2003) and

Received September 26, 2013.

Accepted December 26, 2013.

${ }^{1}$ Corresponding author: damoore@vetmed.wsu.edu farm productivity reasons (Hernandez et al., 2001; Juarez et al., 2003; Cha et al., 2010). Identification of individual cows with lameness is important for delivery of prompt treatment as well as for monitoring levels of disease in a herd. The high prevalence of lameness in dairy herds, over 20\% in some studies (Cook, 2003; Espejo et al., 2006; von Keyserlingk et al., 2012), combined with the finding that producers tend to underestimate lameness within their herds (Wells et al., 1993; Espejo et al., 2006) suggests that methods to easily identify lame cows for treatment would be useful to reduce the number of cows afflicted with this disease. Because prevalence is a function of the duration of a disease as well as its incidence, it is reasonable that faster detection and treatment would decrease duration and, therefore, prevalence. Leach et al. (2012) tested an "early threshold" protocol for lameness detection and treatment, in which all cows were observed for lameness every $14 \mathrm{~d}$ and treated within $48 \mathrm{~h}$ if they had a mobility score of "mildly lame" or greater severity. This protocol resulted in a decreased prevalence of lameness compared with groups of cows in which lameness was detected using existing farm protocols.

Subjective methods of lameness detection include locomotion scoring systems, which require observation of gait characteristics as cows are walking (Flower and Weary, 2009). However, frequent observation of individual cows walking may be difficult on large farms. A more convenient method for detecting lameness would be useful to increase the rate of detection. A common practice on dairies is to lock cows in stanchions on a regular basis for estrus detection, AI, pregnancy diagnosis, and preventive health procedures. This is a time when producers, employees, and veterinarians are already examining individual cows and when the assessment of individual cows for lameness might be most convenient.

Certain postures have been associated with lameness in dairy cattle, some of which can be observed while the cow is standing. Locomotion scoring systems, which have been validated by correlation with hoof and leg pathology (Flower and Weary, 2006; Bicalho et al., 2007; Tadich et al., 2010), often include assessment of 
back posture as a criterion for lameness. In one commonly used scoring system (Sprecher et al., 1997), the nonlame cow (locomotion score $\leq 2$ ) stands with a flat, level back, whereas the "lame" cow (locomotion score $\geq 3$ ) stands with an arched back. The arched-back posture has been associated with sole ulcers (Flower and Weary, 2006) and other hoof lesions (O'Callaghan et al., 2003). O'Callaghan et al. (2003) found that cows with hoof lesions classified as chronic (sole ulcer, white line disease) were more likely to have greater severity of back-arch score compared with cows with hoof lesions classified as acute (digital dermatitis, foot rot), and that more cows with severe hoof lesions had increased back-arch severity score compared with cows with mild hoof lesions. Other postures that have been suggested to be associated with lameness or hoof overgrowth include a cow-hocked stance (in which the points of the hocks are medially directed and the claws of the hind hoof are laterally directed), widely placed hind limbs, and favoring a limb while standing. Toussaint-Raven (1985) suggested that cow-hocks and wide stances are indicative of pain in the lateral claw, which is the claw most commonly affected by hoof disease. Additionally, favoring, or lifting, a limb may be observed while a cow is standing. Leach et al. (2009) developed a system for assessing lameness in tiestall-confined cows that included observation of repeated limb shifting, resting one foot more than the other, uneven weight bearing when encouraged to move from side to side, and reluctance to bear weight on a particular foot. Locomotion scoring systems include reduced weight-bearing on a limb as a criterion for severe lameness (Sprecher et al., 1997; Cook, 2003; Flower and Weary, 2006).

Evaluation of several of these postures has been utilized instead of locomotion scoring as an indicator for lameness. In a trial of foot bathing for treatment of hoof diseases, Randhawa et al. (2008) used cow-hocked hind-leg conformation as a treatment outcome and found a $50 \%$ reduction in the number of cows exhibiting this posture after treatment compared with cows that received no treatment. Zurbrigg et al. (2005) evaluated both back arch and presence of hind-claw lateral rotation $\left(>20^{\circ}\right)$ as an indicator of animal welfare based on their suggested association with lameness. However, hind-limb postures have not been evaluated for association with locomotion score, which is a validated and commonly used method of lameness determination.

Back arch has been evaluated previously for association with lameness. O'Callaghan et al. (2003) found a correlation between spine curvature and locomotion score. Thomsen (2009) evaluated the test characteristics of back arch as a test for lameness determined by locomotion scoring, but cows in that study were assessed for back arch while standing loose in pens, not locked in stanchions. The objectives of the current study were to test the hypothesis that back arch and 3 hind-limb postural abnormalities observed while cows are locked in stanchions are associated with higher locomotion score and, second, to evaluate the sensitivity and specificity of these postural observations, with locomotion scoring as the gold standard.

\section{MATERIALS AND METHODS}

\section{Cow Enrollment and Scoring}

This study was approved by the Washington State University animal care and use committee (Pullman, WA). Cows from 4 farms that volunteered to participate in a lameness reduction project were used for this study; 3 farms were in Washington State and 1 in Oregon. Data were collected in May and June 2011.

Cows were locomotion-scored as they exited the milking parlor or walked freely in their pens, in person, by one of the authors (DM, AH, or JV), who completed the same training using online materials (Zinpro Corp., 2010). The locomotion scoring system was based on Sprecher et al. (1997), where a score of 1 indicates a sound cow with a level back while walking and standing; a score of 2 indicates a cow with an arched-back posture while walking but not while standing; a score of 3 indicates the cow maintains an arched-back posture walking and standing and additionally short-strides one or more legs; a score of 4 indicates the cow additionally has reduced weight-bearing on at least one limb; and a score of 5 indicates refusal to bear weight on a limb. Lameness was defined as locomotion score $\geq 3$ for analysis. Interobserver agreement for locomotion scoring in the present study is described elsewhere (Hoffman et al., 2013).

Cows were locked in stanchions at regularly scheduled times for management (AI, estrus detection, or pregnancy diagnosis). Cows were observed as they stood in stanchions by 1 of 2 of the authors (AH or DM) for back arch and for hind-limb posture viewed from behind. All postural abnormalities were recorded as present or absent. Observed postural abnormalities included arched back, cow-hocks, wide stance, and favoring a limb. A cow was considered to have an arched back if her back was arched upward between the withers (dorsal spinous processes of the first few thoracic vertebrae) and tailhead (sacrococcygeal joint). Cows were only observed for back arch when the head was in a neutral or down position and the cow was not pulling back against her head restraint, defecating, or urinating. Percentage interobserver agreement and kappa (Fleiss et al., 2003) was assessed for back arch using photographs of cows locked in stanchions. A cow was considered to be cow- 
hocked if the hocks were rotated medially such that the point of the hock was directed medially and the lateral claw could be seen directed laterally when viewed from behind. A cow was considered to have a wide stance if the lateral claw was lateral to a vertical line drawn from the tuber coxae to the ground. A cow was considered to be favoring a limb if any part of a hind or fore hoof was elevated from the ground at the time of observation. Cows with full placement of the hooves on the ground were not observed further for shifting weight or development of favored limb as they continued to stand. Data on DIM and parity were obtained from computerized herd records. Herd number, observer, and breed were recorded for each cow. Individual cows were not included in the study if they were not observed for locomotion score because they moved past observers at a gait faster than a walk when exiting the milking parlor or were not observed for posture because they were not locked in stanchions at the regularly scheduled time.

\section{Statistical Analysis}

The $\chi^{2}$ test was used to evaluate the association between the presence of each posture and the classification of lameness (locomotion score $\geq 3$ ). The $\chi^{2}$ test for trend was used to assess the relationship between presence of postural abnormalities and increasing locomotion score. The relationship between lameness and each posture was stratified by parity, observer, breed, herd, and DIM group, and was analyzed using $\chi^{2}$ for differing odds ratios by strata to evaluate confounding and any changes in the relationship between posture and lameness within different risk groups. A logistic regression model was created to assess the relationship between lameness and back arch while controlling for lameness risk factors. The $\chi^{2}$ test for trend, $\chi^{2}$ for differing odds ratios by strata, and logistic regression were performed using statistical software (Epi Info version 3.5.3, Centers for Disease Control and Prevention, Atlanta, GA). Variables included back arch (present or absent), herd, breed (Holstein, Jersey, or Holstein-Jersey cross), parity $(1,2$, or $\geq 3)$, observer (1 or 2$)$, and DIM (1 to 100, 101 to 200,201 to 300,301 to 400 , or $>400$ ), with lameness (locomotion score $\geq 3$ ) as the outcome variable. The final logistic regression model was determined using backward selection. Interactions between variables were assessed. Herd was not included as a variable because it was highly correlated with breed. Variables with $P>$ 0.10 were dropped from the model. For the $\chi^{2}$ test for trend, locomotion scores 4 and 5 were combined such that locomotion score categories were $1,2,3$, and $4+5$. For binomial analyses and logistic regression, lameness was defined as locomotion score $\geq 3$.
To assess the potential usefulness of postural abnormalities for lameness detection, sensitivity, specificity, predictive values, and likelihood ratios were calculated for presence of postural abnormalities as a test for lameness, defined as a locomotion score $\geq 3$. Sensitivity was calculated as the number of lame cows that were observed to be exhibiting a posture divided by the total number of cows classified as lame. Specificity was calculated as the number of nonlame cows (locomotion score $\leq 2)$ that were not exhibiting a posture at the time of observation divided by the total number of nonlame cows. Positive predictive value was calculated as the number of lame cows that were observed to be exhibiting a posture divided by the total number of cows that were observed to be exhibiting that posture. Negative predictive value was calculated as the number of nonlame cows that were not exhibiting a posture at the time of observation divided by the total number of cows that were not exhibiting that posture at observation. Likelihood ratio positive was calculated as (sensitivity)/(1 - specificity) and likelihood ratio negative was $(1-$ sensitivity)/(specificity). These test characteristics were also calculated for combinations of postures as a test for lameness using the postures with the highest sensitivity and specificity combinations.

\section{RESULTS}

\section{Lameness Prevalence and Interobserver Agreement}

Locomotion score and posture data were obtained for 1,243 cows. Cows for which locomotion score and postures while standing were both observed were included in the present study; therefore, cows that did not lock in stanchions or that were not locomotion-scored because they passed observers at a gait faster than a walk were not included. Farm 1 had 148 lactating Holstein cows, all pens were routinely locked in stanchions, and 101 cows were included in the study. Farm 2 had 492 lactating and nonlactating Jersey cows, 2 of 4 pens of lactating cows and 1 pen of nonlactating cows were locked in stanchions daily, and 183 cows were included in the study, including 25 nonlactating cows. Farm 3 had 584 lactating Holstein, Jersey, and Holstein-Jersey crossbreed cows in 4 pens, all of which were locked in stanchions daily, and 286 cows were included in the study. Farm 4 had 1,131 lactating Holstein cows in 9 pens, 8 pens were locked in stanchions daily, and 673 cows were included in the study. Overall lameness prevalence among these, determined by locomotion scoring, was $37,11,17$, and $34 \%$ for herds 1 through 4 , respectively. Back-arch observation agreement between the 2 observers was $75 \%$ and kappa was 0.4 . 
Table 1. Final logistic regression model for back arch and factors associated with lameness in 1,243 lactating dairy cattle

\begin{tabular}{llcccc}
\hline & & & \multicolumn{2}{c}{$95 \%$ CI } & \\
\cline { 5 - 6 } Item & Term & Odds ratio & Lower & Upper & P-value \\
\hline Posture & Back arch & 2.5 & 1.9 & 3.4 & $<0.001$ \\
Breed & Holstein & Referent & & & \\
& Jersey & 0.36 & 0.21 & 0.60 & $<0.001$ \\
\multirow{2}{*}{ DIM } & Cross & 0.44 & 0.17 & 1.1 & 0.09 \\
& 1 to 100 & Referent & & & \\
& 101 to 200 & 1.1 & 0.75 & 1.6 & 0.66 \\
& 201 to 300 & 2.0 & 1.37 & 2.94 & $<0.001$ \\
& 301 to 400 & 1.99 & 1.26 & 3.16 & 0.003 \\
Parity & $>400$ & 3.94 & 1.96 & 7.92 & $<0.001$ \\
& 1 & Referent & & & \\
& 2 & 2.8 & 1.9 & 4.1 & $<0.001$ \\
& $\geq 3$ & 6.3 & 4.4 & 9.1 & $<0.001$ \\
\hline
\end{tabular}

\section{Association Between Postures and Locomotion Score}

Back Arch. Observation of back arch was associated with locomotion score $\geq 3(P<0.001)$. Cows with an arched-back posture had 3 times greater odds of being lame (locomotion score $\geq 3$ ) compared with cows without an arched back (odds ratio $=3,95 \%$ CI: 2.3-3.9). The $\chi^{2}$ test for trend showed an association between increasing locomotion score and presence of a back arch $(P<0.001 ;$ Figure 1). We detected a trend for difference in the strength of association between back arch and lameness by parity $(P=0.1)$ and observer $(P=$ $0.07)$ in the $\chi^{2}$ test for differing odds ratio by strata. The strength of association was greater within parity group $\geq 3$ and for observer 2 . We found no differences in the strength of association between locomotion score $\geq 3$ and back-arch observation by breed, herd, or DIM group $(P>0.1)$. In the final logistic regression model, back arch was associated with lameness (Table 1). Because breed and herd were correlated, only breed was entered into the model. Breed, DIM group, and parity were retained as variables, whereas observer was not significantly associated with the observation of lameness.

Cow-Hocked Posture. Observation of cow-hocked posture was associated with lameness $(P<0.001)$. Cows with a cow-hocked posture were 1.6 times $(95 \%$ CI: $1.2-2.0$ ) more likely to be lame (locomotion score $\geq 3$ ) compared with cows without cow-hocked posture. The $\chi^{2}$ test for trend showed an increasing proportion of observed cow-hocked posture with increasing locomotion score $(P<0.001)$. We detected no differences by breed, DIM group, parity, herd, or observer for cowhocked posture $(P>0.1)$.

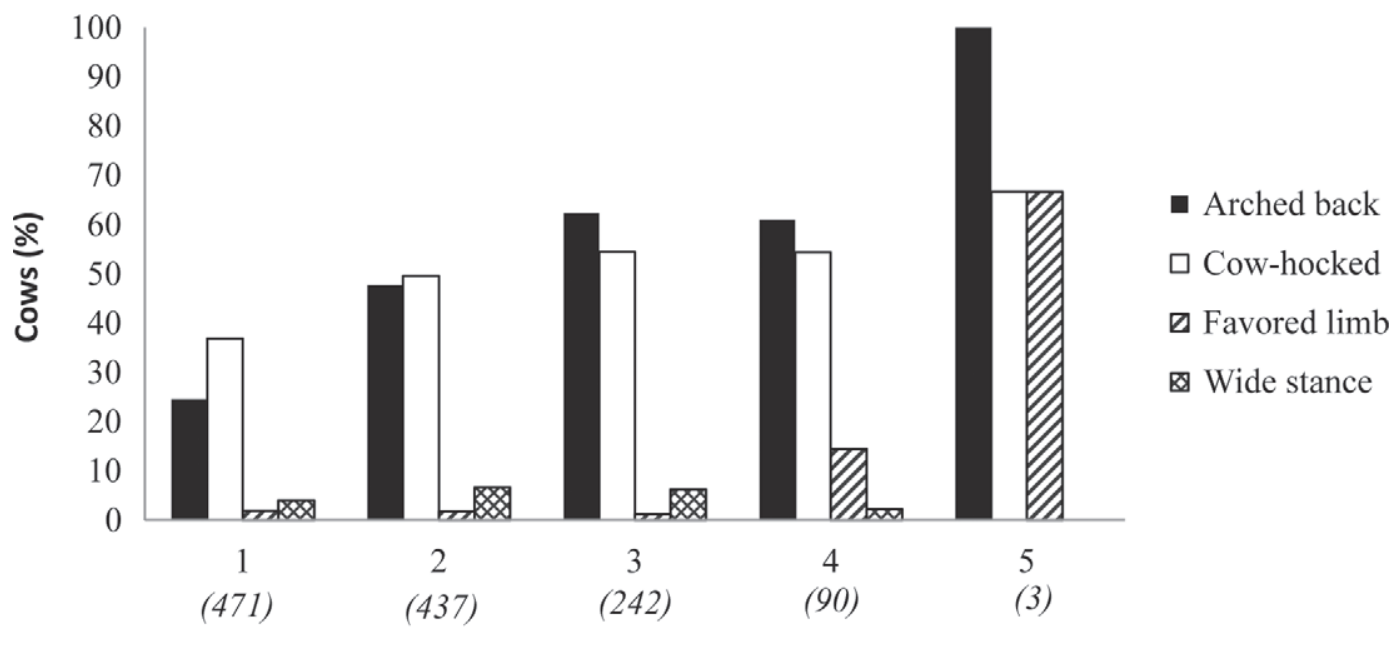

Locomotion Score (Number of cows)

Figure 1. Percentage of 1,243 lactating dairy cows with arched back and cow-hocked, favored-limb, and wide-stance postures observed when cows were standing at the stanchions for each locomotion score assigned when observed walking. Numbers represent total number of cows in each locomotion score category. 
Table 2. Test characteristics for presence of postural abnormalities or combinations of postural abnormalities as a test for lameness (locomotion score $\geq 3$ ) in 1,243 lactating dairy cattle ${ }^{1}$

\begin{tabular}{lcccccc}
\hline Posture or combination & Sensitivity & Specificity & PPV & NPV & LR positive & LR negative \\
\hline Back arch & 0.63 & 0.64 & 0.39 & 0.82 & 1.75 & 0.58 \\
Cow-hocks & 0.54 & 0.57 & 0.32 & 0.77 & 1.26 & 0.81 \\
Favored limb & 0.05 & 0.98 & 0.51 & 0.74 & 2.50 & 0.97 \\
Back arch or cow-hocks & 0.78 & 0.40 & 0.33 & 0.84 & 1.30 & 0.55 \\
Back arch and cow-hocks & 0.39 & 0.81 & 0.42 & 0.78 & 1.99 & 0.76 \\
\hline
\end{tabular}

${ }^{1} \mathrm{PPV}=$ positive predictive value; $\mathrm{NPV}=$ negative predictive value $\mathrm{LR}=$ likelihood ratio.

Favored-Limb Posture. Favored-limb posture was associated with lameness $(P<0.005)$. Cows favoring a limb were almost 3 times more likely to be lame $(95 \%$ CI: 1.5-5.9) compared with cows not observed favoring a limb. We detected no differences in the association between observation of favored-limb posture and lameness by breed, DIM group, parity, or herd $(P>0.1)$. We did detect a trend for difference by observer $(P=$ $0.1)$.

Wide-Stance Posture. Wide stance was not associated with lameness overall $(P=0.99)$ and we found no association between wide-stance posture and lameness when stratified by breed, DIM group, parity, observer, and herd $(P>0.05)$.

\section{Test Characteristics}

The presence of back arch, cow-hocked posture, favored-limb posture, and combinations of back arch and cow-hocked posture were evaluated as "tests" for lameness (Table 2). Sensitivity was highest for back arch, and the specificity of back arch was higher than specificity of cow-hocked posture (Table 2). The highest specificity was for a favored limb (0.98), but the test sensitivity was only 0.05 .

Sensitivity and specificity of back arch as a test for lameness were calculated for each breed. Back arch in Jersey breed cows as a test for lameness had a lower sensitivity (29\%) and higher specificity (93\%) than overall sensitivity and specificity $(P<0.05)$. Back arch had a higher specificity in Holstein-Jersey crossbreeds $(83 \% ; P<0.05)$ compared with overall specificity. Sensitivity and specificity of back arch was calculated using different locomotion score levels (locomotion score $\geq 2$, $\geq 4$, or $\geq 5$ instead of $\geq 3$ ) as the definition of lameness (Table 3). Sensitivity was not changed by increasing the locomotion score cutoff and was decreased by lowering the locomotion score cutoff below $\geq 3(P<0.05)$. Specificity was higher if locomotion score 2 was used as a cutoff $(P<0.05)$ and lower if locomotion score 4 or 5 was used as the cutoff $(P<0.05)$.

\section{DISCUSSION}

The lameness prevalence in the 4 herds included in this study was 11 to $37 \%$, which is within the range of lameness prevalence previously reported in North American dairy herds, although different methods of scoring and different groups of cows may have been observed (Cook, 2003; Espejo et al., 2006; von Keyserlingk et al., 2012). The kappa statistic for interobserver back-arch agreement was considered indicative of a fair strength of agreement. Although interobserver agreement was not assessed for cow-hocked or favored-limb postures, observer was not found to confound the relationship between these postures and lameness.

In this study, presence of arched-back, cow-hocked, wide-stance, and favored-limb postures observed while cows stood locked in stanchions were assessed for association with locomotion score and lameness (locomotion score $\geq 3$ ). The presence of arched-back, cow-hocked, and favored-limb postures were positively associated with locomotion score. The higher the locomotion score (indicative of a higher degree of gait abnormality), the more likely these postural abnormalities were to be observed. The association of lameness with cow-

Table 3. Sensitivity, specificity, and $95 \%$ CI for back arch posture as a test for lameness with differing locomotion score cutoff points as definition of lameness

\begin{tabular}{lcccc}
\hline $\begin{array}{l}\text { Locomotion } \\
\text { score cutoff }\end{array}$ & Sensitivity & $95 \%$ CI & Specificity & $95 \%$ CI \\
\hline$\geq 2$ & $0.54^{*}$ & 0.51 to 0.58 & $0.71^{*}$ & 0.67 to 0.75 \\
$\geq 3$ & 0.63 & 0.57 to 0.68 & 0.64 & 0.61 to 0.67 \\
$\geq 4$ & 0.62 & 0.52 to 0.72 & $0.59^{*}$ & 0.56 to 0.61 \\
$\geq 5$ & 1 & 0.38 to 1 & $0.57^{*}$ & 0.54 to 0.60 \\
\hline
\end{tabular}

$* P<0.05$ compared with locomotion score $\geq 3$ as a cutoff. 
hocked stance supports the previous suggestion of Toussaint-Raven (1985) that this posture can indicate the presence of hoof pathology. Toussaint-Raven (1985) suggested that cow-hocked stance relieves pressure on the lateral claw, the most common site of lesions, which could explain the lower association between this posture and lameness than between back arch and lameness if only cows with lateral claw pathology exhibit this posture. Cow-hocked stance has been evaluated in genetic and phenotypic studies of locomotion. Rear leg rear view conformation scores (where direction of the hocks is evaluated) were found to be genetically and phenotypically associated with locomotion and phenotypically positively associated with claw disorders (van der Waaij et al. 2005). The association between cow-hocked stance and locomotion score in the present study could be partly or entirely due to inherited cowhocked stance predisposing to lameness, rather than cow-hocked stance being a clinical sign of lameness. Wide stance, overall, was not found to be associated with locomotion score in this study. This is likely due to other reasons causing the wide stance that are not associated with lameness, such as large udder size.

The association between back arch and locomotion score has been established by others (O'Callaghan et al., 2003; Flower and Weary 2006), and the present study demonstrates that back arch can be observed as cows stand in stanchions and maintain this association with locomotion score. The observation of a favored limb was associated with lameness, consistent with reduced weight-bearing associated with limb pain. The proportion of cows observed favoring a limb was highest for locomotion scores 4 and 5, where reduced weightbearing is part of the criteria for defining these scores. However, relatively few cows were observed favoring a limb compared with other postures.

Presence of these postures was also evaluated as a "diagnostic test" for locomotion score $\geq 3$. The specificity of back arch in the present study was lower than that reported by Thomsen (2009), who found a specificity of $86 \%$ for back arch as a test for lameness. Thomsen (2009) assessed cows as they stood freely in pens, not locked in stanchions, which could be responsible for the higher false-positive rate in the present study, because cows with their heads in a stanchion or in the down position while eating may have an increased arch in the spine. Additionally, the angle at which spine curvature is observed could confound comparison of our current results with those of Thomsen (2009). The observation of cows loose in the pens would allow the observer to assess back arch while viewing the cow laterally, at a $90^{\circ}$ angle to the spine, whereas in the present study (viewing each cow from a caudal direction), back arch could only be assessed from an angle of $45^{\circ}$ or less to the spine. This difference in observation angle could alter the observer's evaluation of spine curvature, although neither Thomsen (2009) nor the present study evaluated the effect of observation angle on the detection of back arch. The locomotion scoring system used by Thomsen (2009) and that used herein are very similar, both being based on criteria developed by Sprecher et al. (1997). However, Thomsen (2009) used locomotion score $\geq 4$ as a cutoff for lameness. Locomotion scores 3 and 4 are both described as standing with an arched back, but a score of 4 has a more severely affected limb. In the present study, we found no difference in test characteristics of arched back when using score 3 or score 4 as a locomotion score cutoff point for defining lameness. Another difference between these 2 studies is that Thomsen (2009) evaluated only Holstein cows, whereas Jersey and Holstein-Jersey crossbreeds were included in the present study. However, back arch in Jersey cows in the current study had a higher specificity and lower sensitivity than in Holsteins, similar to the test characteristics established by Thomsen (2009).

Another possibility for the level of false positives for back arch as a test for lameness in the present study is the discrepancy in the clinical signs of individual cows noted for classification of locomotion scores. The definition of locomotion score 3 (the cutoff point for classification as lame) used in the present study was that the cow walked and stood with an arched back and exhibited a short striding gait. If the cow was not seen to be standing when locomotion scoring was performed, an arched back and short striding gait were sufficient to define the cow as a 3 , whereas if she was seen walking with an arched back and no short striding gait, she was classified as a 2. It is unknown how many cows both walk and stand with an arched back but do not have a short striding gait. This presentation could result in a false positive if assessing back arch alone as a test for lameness in cows that have a back arch but not a short-stride gait. In addition, arched back has been described as a clinical sign of disease syndromes other than lameness, including abdominal pain, pericardial disease, and pleuropneumonia (Radostits et al., 2007). Cows exhibiting an arched back for other reasons would be misclassified as lame by back-arch observation alone.

Cows that walk with an arched back and short stride but do not stand with an arched back would be classified as lame by locomotion scoring and as nonlame by observation of back arch alone, resulting in a false negative. Sprecher et al. (1997) classified a locomotion score 3 as both short-striding and standing with an arched back, whereas Thomsen (2009) classified a score 3 and score 4 as, in most cases, the back being arched both standing and walking. The appropriate locomotion score cutoff for allocation of lame and nonlame cows 
may differ for farm management purposes (detection of cows for treatment) versus research or welfare assessment purposes (overall prevalence of this clinical sign of hoof and leg disease at varying stages). Although locomotion score 3 has been used previously as a definition for lameness in published research (Espejo et al., 2006; von Keyserlingk et al., 2012), other locomotion scoring systems and cutoff levels have also been used (Cook, 2003; Leach et al., 2012). Clarity and consistency of language used to define locomotion scores is needed to increase accuracy and precision of locomotion scoring systems as well as aid in useful comparisons among research studies. Future research may further define the usefulness of back arch as an indicator of lameness and the appropriate scoring system to be used as a gold standard.

In regard to on-farm utility of back arch as an indicator of lameness, an important consideration is the likelihood of finding cows with painful hoof lesions to treat. Increased locomotion score has been associated with increased proportion with painful hoof lesions, but in one study (Bicalho et al., 2007), only $55.5 \%$ of locomotion score 3 cows had painful hoof lesions. The same study found that the sensitivity and specificity of locomotion scoring, using painful hoof lesions as the gold standard and locomotion score 3 as the cutoff for defining lameness, were 67.5 and $84.6 \%$, respectively. Considering the sensitivity and specificity of back arch for detecting locomotion score $\geq 3$ and the sensitivity and specificity of locomotion score $\geq 3$ for detecting painful lesions, a higher likelihood exists of detecting cows with hoof pathology among those that stand with an arched back in stanchions than among those that do not. It may not be economically feasible to examine the hooves of every cow with an arched back, but it may be useful to use observation of back arch in stanchions as a screening tool to be followed by locomotion scoring and hoof examination in those determined to be lame. In this way, the observation of back arch as cows stand in stanchions could increase the rate of early lameness detection and treatment.

\section{CONCLUSIONS}

Although the present results for test characteristics of various postures as a test for lameness were not acceptable by many diagnostic standards, they may have utility for on-farm disease screening. According to the results of the present study, this test is not sensitive and specific enough to identify individual lame cows or to calculate herd prevalence. However, because lame cows are more likely to exhibit these postures, their observation may be useful for screening cows during routine management times as an aid in lameness detec- tion. Further research could clarify the source of false positives and false negatives of back arch as a test for lameness.

\section{ACKNOWLEDGMENTS}

This project was supported by the Washington State Dairy Federation (Elma, WA) and Washington Dairy Products Commission (Lynnwood, WA). The authors thank the collaborating dairy producers and veterinarians Fred Muller (Sunnyside, WA), Robert Holt and Jacob Steiger (Lynden, WA), and Mike Anderson and Gale DeJong (Lynden, WA) for sharing their valuable time and input to the project.

\section{REFERENCES}

Bicalho, R. C., S. H. Cheong, G. Cramer, and C. L. Guard. 2007. Association between a visual and an automated locomotion score in lactating Holstein cows. J. Dairy Sci. 90:3294-3300.

Cha, E., J. A. Hertla, D. Barb, and Y. T. Gröhn. 2010. The cost of different types of lameness in dairy cows calculated by dynamic programming. Prev. Vet. Med. 97:1-8.

Cook, N. B. 2003. Prevalence of lameness among dairy cattle in Wisconsin as a function of housing type and stall surface. J. Am. Vet. Med. Assoc. 223:1324-1328.

Espejo, L. A., M. I. Endres, and J. A. Salfer. 2006. Prevalence of lameness in high-producing Holstein cows housed in freestall barns in Minnesota. J. Dairy Sci. 89:3052-3058.

Fleiss, J. L., B. Levin, and M. C. Paik. 2003. Statistical Methods for Rates and Proportions. John Wiley and Sons, New York, NY.

Flower, F. C., and D. M. Weary. 2006. Effect of hoof pathologies on subjective assessments of dairy cow gait. J. Dairy Sci. 89:139-146.

Flower, F. C., and D. M. Weary. 2009. Gait assessment in dairy cattle. Animal 3:87-95.

Hernandez, J. A., J. K. Shearer, and D. W. Webb. 2001. Effect of lameness on the calving-to-conception interval in dairy cows. J. Am. Vet. Med. Assoc. 218:1611-1614.

Hoffman, A. C., D. A. Moore, J. R. Wenz, and J. Vanegas. 2013. Comparison of modeled sampling strategies for estimation of dairy herd lameness prevalence and cow-level variables associated with lameness. J. Dairy Sci. 96:5746-5755.

Juarez, S. T., P. H. Robinson, E. J. DePeters, and E. O. Price. 2003 Impact of lameness on behavior and productivity of lactating Holstein cows. Appl. Anim. Behav. Sci. 83:1-14.

Leach, K. A., S. Dippel, J. Huber, S. March, C. Winckler, and H. R. Whay. 2009. Assessing lameness in cows kept in tie stalls. J. Dairy Sci. 92:1567-1574.

Leach, K. A., D. A. Tisdall, N. J. Bell, D. C. J. Main, and L. E. Green. 2012. The effects of early treatment for hindlimb lameness in dairy cows on four commercial UK farms. Vet. J. 193:626-632.

O'Callaghan, K. A., P. J. Cripps, D. Y. Downham, and R. D. Murray. 2003. Subjective and objective assessment of pain and discomfort due to lameness in dairy cattle. Anim. Welf. 12:605-610.

Radostits, O. M., C. C. Gay, K. W. Hinchcliff, and P. D. Constable. 2007. Veterinary Medicine: A Textbook of the Diseases of Cattle, Horses, Sheep, Pigs and Goats. 10th ed. Saunders, London, UK.

Randhawa, S. S., K. Dua, P. S. Dhaliwal, S. K. Uppal, and S. T. Singh. 2008. Effect of formalin footbathing on the prevalence of foot lesions and conformational indices in dairy cattle. Vet. Rec. 163:335-337.

Sprecher, D. J., D. E. Hostetler, and J. B. Kaneene. 1997. A lameness scoring system that uses posture and gait to predict dairy cattle reproductive performance. Theriogenology 47:1179-1187.

Tadich, N., E. Flor, and L. Green. 2010. Associations between hoof lesions and locomotion score in 1098 unsound dairy cows. Vet. J. 184:60-65. 
Thomsen, P. T. 2009. Rapid screening method for lameness in dairy cows. Vet. Rec. 164:689-690.

Toussaint-Raven, E. 1985. Cattle Footcare and Claw Trimming. Farming Press, Ipswich, UK.

van der Waaij, E. H., M. Holzhauer, E. Ellen, C. Kamphuis, and G. de Jong. 2005. Genetic parameters for claw disorders in Dutch dairy cattle and correlations with conformation traits. J. Dairy Sci. 88:3672-3678.

von Keyserlingk, M. A. G., A. Barrientos, K. Ito, E. Galo, and D. M. Weary. 2012. Benchmarking cow comfort on North American freestall dairies: Lameness, leg injuries, lying time, facility design, and management for high-producing Holstein dairy cows. J. Dairy Sci. 95:7399-7408.
Wells, S. J., A. M. Trent, W. E. Marsh, and R. A. Robinson. 1993. Prevalence and severity of lameness in lactating cows in a sample of Minnesota and Wisconsin herds. J. Am. Vet. Med. Assoc. 202:78-82.

Zinpro Corp. 2010. Locomotion scoring of dairy cattle. Zinpro Corp., Eden Prairie, MN. Accessed May 10, 2011. http://www.zinpro. com/video-library/dairy-locomotion-videos.

Zurbrigg, K., D. Kelton, N. Anderson, and S. Millman. 2005. Tie-stall design and its relationship to lameness, injury, and cleanliness on 317 Ontario dairy farms. J. Dairy Sci. 88:3201-3210. 